\title{
Identification of MicroRNA-Like Molecules Derived from the Antigenome RNA of Hepatitis C Virus: A Bioinformatics Approach
}

\author{
Juan Cristina*, Ricardo Recarey \\ Laboratorio de Virología Molecular, Centro de Investigaciones Nucleares, Facultad de Ciencias, Universidad de \\ la República, Montevideo, Uruguay \\ Email: "cristina@cin.edu.uy, rrecarey@cin.edu.uy
}

Received 31 October 2015; accepted 5 April 2016; published 8 April 2016

Copyright $(2016$ by authors and Scientific Research Publishing Inc.

This work is licensed under the Creative Commons Attribution International License (CC BY). http://creativecommons.org/licenses/by/4.0/

(c) $\underset{\mathrm{EY}}{\mathrm{C}}$ Open Access

\begin{abstract}
MicroRNAs (miRNAs) are small, noncoding RNA molecules that play important roles in the regulation of gene expression of the cell. Recent studies have described cytoplasmic RNA virus genomederived miRNAs. Moreover, miRNAs have also been encountered in the reverse strand of the viral mRNA, revealing the presence of miRNAs in replication intermediaries. In order to get insight into the possible role of Hepatitis C Virus (HCV) antigenome in relation to miRNA coding, we computationally identified potential miRNAs on the antigenome of HCV reference strain $\mathrm{H77}$. By utilizing a series of bioinformatics tools, we identified a miRNA present in the antigenomeof HCV H77 strain. This miRNA maps in the 5'non-translated region (5'UTR) of the HCV genome and is found to be conserved among HCV genotypes and sub-types. In silico target prediction generated 17 cellular genes. These potential targets are involved in apoptosis as well as immune response pathways, suggesting that they could play a role in the pathogenesis caused by viral infection. The results of these studies revealed the presence of a viral miRNA in the negative-sense RNA strand used as a replication template for the HCV genome, as observed for other RNA viruses.
\end{abstract}

\section{Keywords}

Hepatitis C Virus, Antigenome, miRNA, MicroRNA

\section{Introduction}

Hepatitis C virus (HCV) is a positive-polarity, single-stranded RNA virus that belongs to the genus Hepacivirus

"Corresponding author.

How to cite this paper: Cristina, J. and Recarey, R. (2016) Identification of MicroRNA-Like Molecules Derived from the Antigenome RNA of Hepatitis C Virus: A Bioinformatics Approach. Natural Science, 8, 180-191. 
in the family Flaviviridae [1]. More than 130 million people worldwide are persistently infected by HCV and are at risk of developing severe liver disease and hepatocellular carcinoma [2] [3]. HCV has a genome of $9.6 \mathrm{~kb}$ in length flanked by two untranslated regions at the 5' and 3'of the genome (5'-UTR and 3'-UTR). The virus encodes a single polyprotein precursor that is subsequently cleaved into at least 10 different proteins, including the structural core (C) and envelope (E1, E2, and p7) proteins as well as the nonstructural proteins (NS2, NS3, NS4A, NS4B, NS5A, and NS5B) [4]. HCV is classified into 7 genotypes, which are subdivided into 67 epidemiologically diverse subtypes [5]. The main $7 \mathrm{HCV}$ genotypes vary in their geographical distribution and their level of genetic diversity. HCV genetic variability has an important impact on disease epidemiology and clinical practice because the viral genotype may determine the pathogenesis and severity of the resultant chronic liver disease. In addition, there is a clear association between the HCV genotype and its susceptibility to antiviral treatment [6]. At this moment, there is no effective vaccine for the prevention and control of HCV infection.

MicroRNAs (miRNAs) are genomically encoded, small noncoding RNA molecules, generally 18 to 22 base pairs in length, and play a crucial role in the regulation of gene expression [7] [8]. Several reports have shown that some plants, animals and viruses encode the miRNAs to regulate their diverse biological processes, including development, apoptosis, tumorogenesis, proliferation, stress response, etc. [9]. miRNAs encoded by viruses are unique, since they regulate not only their own gene expression but also their host gene expression [10].

Genes encoding miRNAs are transcribed by RNA polymerase II and form transcripts as primary miRNAs. These primary miRNAs are processed by ribonuclease Drosha to produce precursor miRNAs, known as premiRNAs, which is exported into the cytoplasm and cleaved by the ribonuclease Dicer to produce mature, single stranded miRNAs [11]. Once synthesized, mature miRNA binds to two proteins, GW182 and Argonaute/EIF2C (AGO) family proteins and forms a complex called miRNA induced silencing complex (miRISC) and mediate the target mRNA recognition. miRNA regulation takes place at multiple steps, including their transcription, their processing by Drosha and Dicer, their loading onto AGO proteins and miRNA turnover [12].

The complementary between miRNAs and target mRNA triggers gene silencing by the mRNA degradation or translational repression of target mRNA [13].

There are three modes of hybridization pattern between miRNAs and their target sites including 5'canonical, 5'seed and 3'compensatory hybridization. 5'canonical pattern shows at least seven nucleotides base-pairings at 5'-end of miRNA at positions 2 to 8, which is known as the seed region and additional base-pairings at 3'-end. 5'seed pattern also consists of seven nucleotides hybridization at positions 2 to 8 within the 5'-end but no base-pairing at the 3-end. 3'compensatory pattern consists of several base-pairings from the middle of the 3'-end of the miRNAs without base-pairing in the seed region [14].

To date, RNA virus-encoded miRNAs have been identified in Hepatitis C virus [15], Human Immunodeficiency virus (HIV) [16], Bovine Leukemia virus (BLV) [17], West Nile virus (WNV) [18], Dengue virus(DENV) [19] and Middle East Respiratory Syndrome (MERS) coronavirus [20]. Very recent studies revealed that the antigenome of another cytoplasmic RNA virus, Hepatitis A virus (HAV), could be processed into miRNA-like small RNAs by the cellular miRNA processing machinery, similar to virus genomes [21].

In order to get insight into the possible roll of antigenomes of HCV in relation to miRNA coding, we computationally identified potential miRNAs on the antigenome of Hepatitis C virus (HCV) reference strain $\mathrm{H} 77$ (genotype 1a, EMBL accession number: NC_004102). Our study may help to better understand host pathogen interaction as well as to contribute to the development of new antiviral therapies against HCV.

\section{Materials and Methods}

\subsection{Viral Sequences and the Prediction of Pre-miRNAs by an ab Initio Approach}

For this work, we used complete Hepatitis C virus (HCV) reference strain H77 genomic sequences (genotype 1a, EMBL accession number: NC_004102), which included the ORFs and the 5' and 3'-UTRs of the viral RNA. Figure 1 shows a flowchart of the computational prediction process. Complete antigenomic sequences were obtained in silico by reverse complement using software from the MEGA program [22].

VMir program [23] was used to analyze the HCV H77 strain sequences. VMir is an $a b$ initio prediction program specifically designed to identify pre-miRNA in viral genomes. Using this approach, HCV H77 antigenome sequences were analyzed for possible pre-miRNA hairpin structures, using default as well as stringent filtering parameters (minimum hairpin size of 60 nucleotides (nt), maximum hairpin size of $120 \mathrm{nt}$, minimum hairpin score of 115, minimum window count of 25). VMir scores were calculated according to Grundhoff [23]. Only 


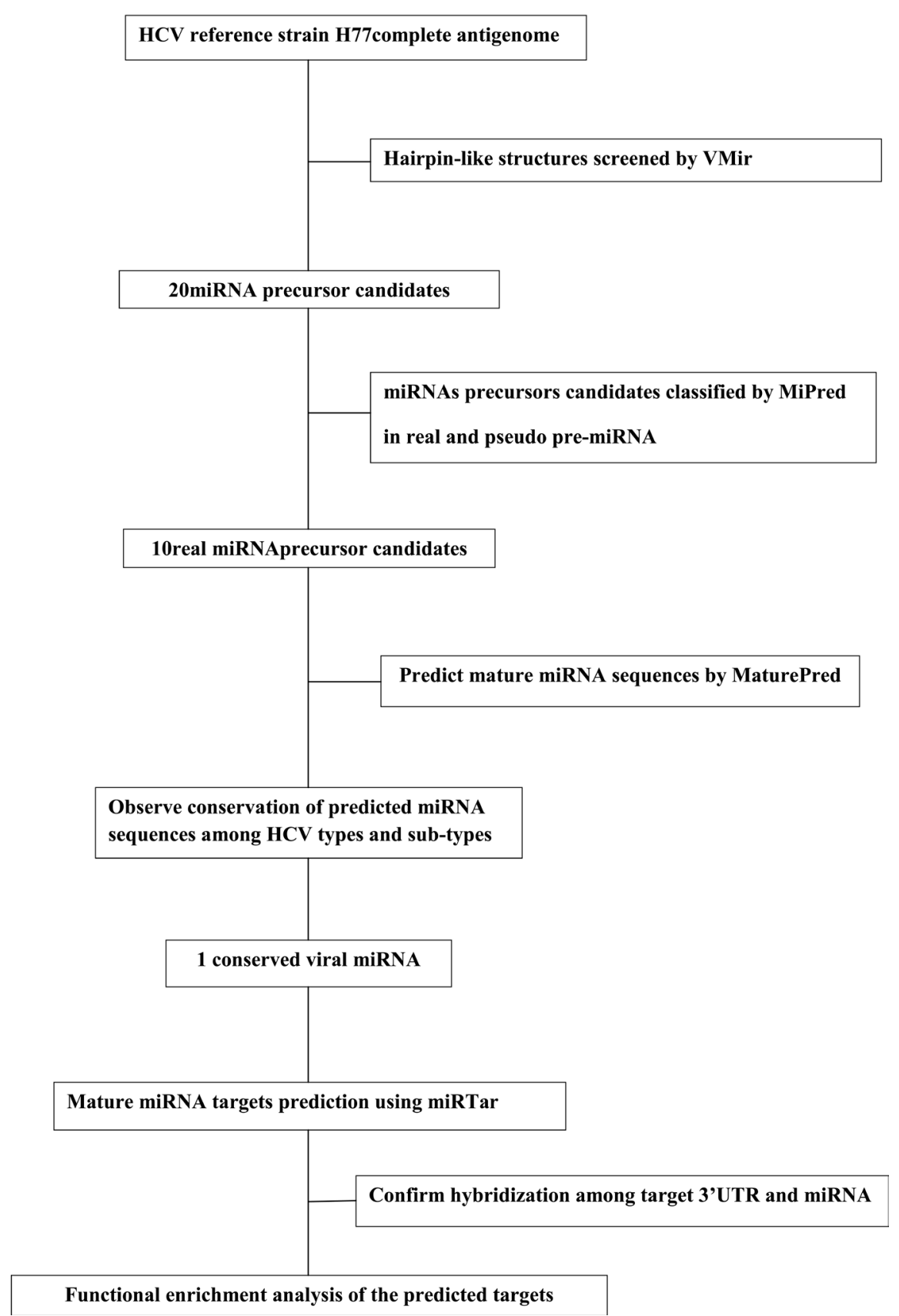

Figure 1. Schematic representation of the methodology employed during miRNA prediction.

pre-miRNA hairpin structures with a minimum score of 140 were considered.

\subsection{Confirmation of Putative Pre-miRNA Sequences}

To discriminate real pre-miRNAs from other hairpin structures (pseudo hairpins) we employed MiPred [24] (available at: http://www.bioinf.seu.edu.cn/miRNA/), which decides whether each hairpin is either a pseudo-premiRNA-like hairpin or a real pre-miRNA using random forest prediction model.

\subsection{Identification of Mature miRNA Sequences}

With the purpose of extracting mature miRNA: miRNA* duplexes from pre-miRNA hairpins, we employed the 
MaturePred Web Tool (available at: http://nclab.hit.edu.cn/maturepred/). This software uses a model based on Support Vector Machine (SVM) that predicts the starting position of an miRNA by performing discriminant analysis against the query hairpin structure using various features of known real/pseudo miRNA: miRNA* duplexes as a training set (position-specific features, energy-related features, structure-related features, and stabilityrelated features) [25].

\subsection{Conservation of Predicted miRNA Sequences among HCV Genotypes and Subtypes}

Similarity among predicted miRNA sequences in HCV H77 strain (genotype 1a) and corresponding sequences of all HCV types and sub-types isolated elsewhere were observed by means of the use of a curated reference alignment (which includes all HCV types and subtypes), obtained from the HCV Database (available at: http://hcv.lanl.gov/content/sequence/NEWALIGN/align.html) [26].

\subsection{Prediction of Secondary Structure of miRNA Precursors}

The RNAfold web server [27] (available at http://rna.tbi.univie.ac.at/cgi-bin/RNAfold.cgi) was used to predict the secondary structure of pre-miRNAs. Only default parameters were used. In all cases, folding structures with centroid were depicted.

\subsection{Prediction of Potential Targets and Functional Enrichment Analysis}

In order to identify the regulatory relationships between predicted miRNAs and their targets gene transcripts we employed miRTar (available at: http://mirtar.mbc.nctu.edu.tw) [28]. The parameters assigned for the hybridization were set to $-14 \mathrm{kcal} / \mathrm{mol}$ of minimum free energy (MFE) for all hybridizations as a cut-off value and an alignment score $\geq 140$. We study the relation among predicted miRNAs and their targets 3'untranslated regions (3UTR) gene transcripts involved in six different metabolic pathways (apoptosis, chemokine signaling pathway, cytokine-cytokine receptor interaction, Jak-STAT signaling pathway, mTOR signaling pathway and T cell receptor signaling pathway). In order to enrich the identified genes with connection to specific functional terms, the potential targets were analyzed using KEGG pathway maps from miRTar [28] to elucidate the biological roles of miRNAs in biological pathways. The $p$-values of the miRNA target genes in the metabolic pathway were also determined. Only genes with a $p$-value $\leq 0.05$ were included in the analysis.

\subsection{Hybridization between 3'UTR Gene Transcripts Target and Mature HCV miRNA}

In order to confirm an effective hybridization between 3'UTR gene transcript targets and predicted HCV miRNA, we employed the RNAhybrid tool [29] (available at: http://bibiserv2.cebitec.uni-bielefeld.de/rnahybrid/). RNA hybrid is a tool for finding the minimum free energy hybridization of a long and a short RNA and widely used for miRNA target prediction. Pairing energy or minimum free energy (MFE) indicates the stability of the hybridization. The MFE was set at $-14 \mathrm{kcal} / \mathrm{mol}$ for all hybridizations, as a cut-off value.

\section{Results}

\subsection{Prediction of Pre-miRNA Stem-Loop Structures in Hepatitis C Virus Antigenome}

Computational prediction represents a widely used and effective strategy to identify novel miRNAs that can be further examined and validated by experimental approaches. RNA virus antigenomes are present in the cytoplasm and form various secondary structures that constitute potential pre-miRNAs. In order to observe whether HCV antigenome could be folded into putative pre-miRNA stem-loop structures, we first analyzed its putative miRNA-encoding capacity using full-length antigenome sequences of HCV H77 reference strain were screened (Figure 2). As default settings were used, 251 candidate hairpins (Figure 2(a)) were identified. To avoid bona fide pre-miRNA hairpin, we filtered VMir output using custom setting, establishing a cut-off value of 60 nucleotides (nt) for minimum hairpin size, $120 \mathrm{nt}$ for maximum hairpin size, and 115 minimum hairpin score. These settings revealed 82 pre-miRNA hairpins (Figure 2(b)) that were selected as potential hairpins. These results suggested the potential miRNA-encoding capacity of the antigenome of this HCV strain.

From these 82 potential pre-miRNAs, potential stem-loop structures with VMir scores higher of 140 were selected for further analysis. Then, in order to confirm the presence of these pre-miRNA structures, we classified 


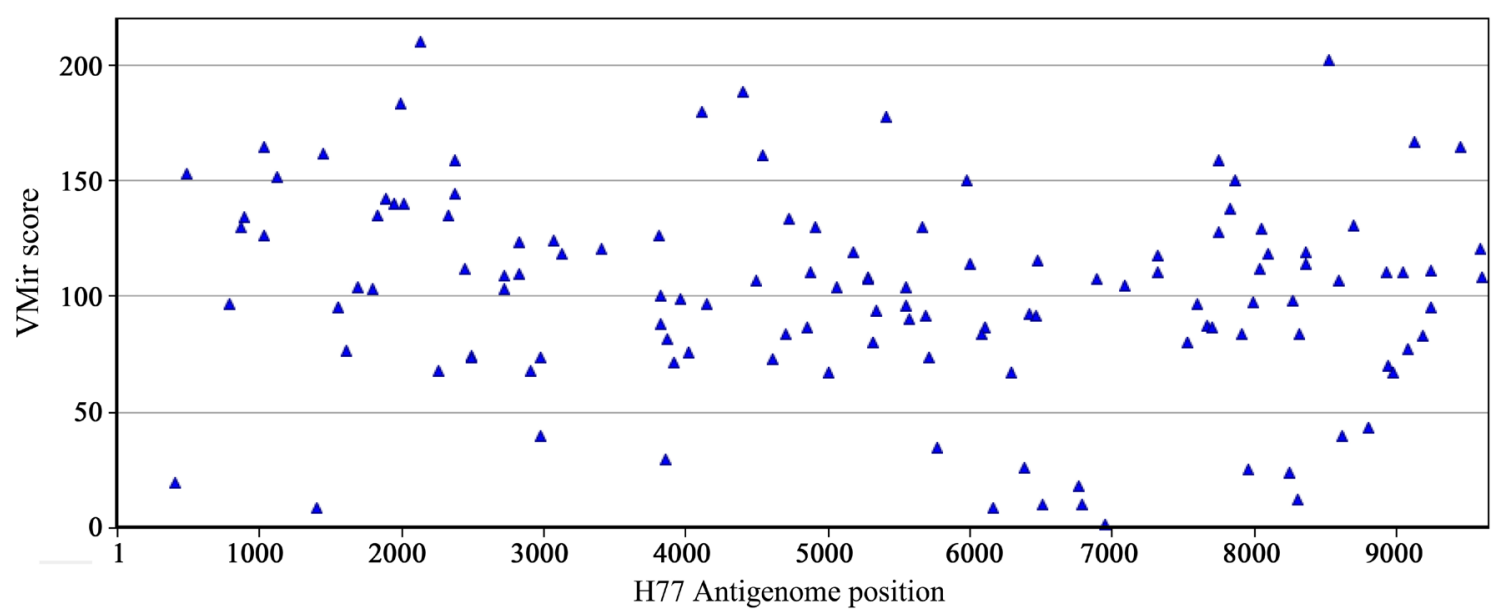

(a)

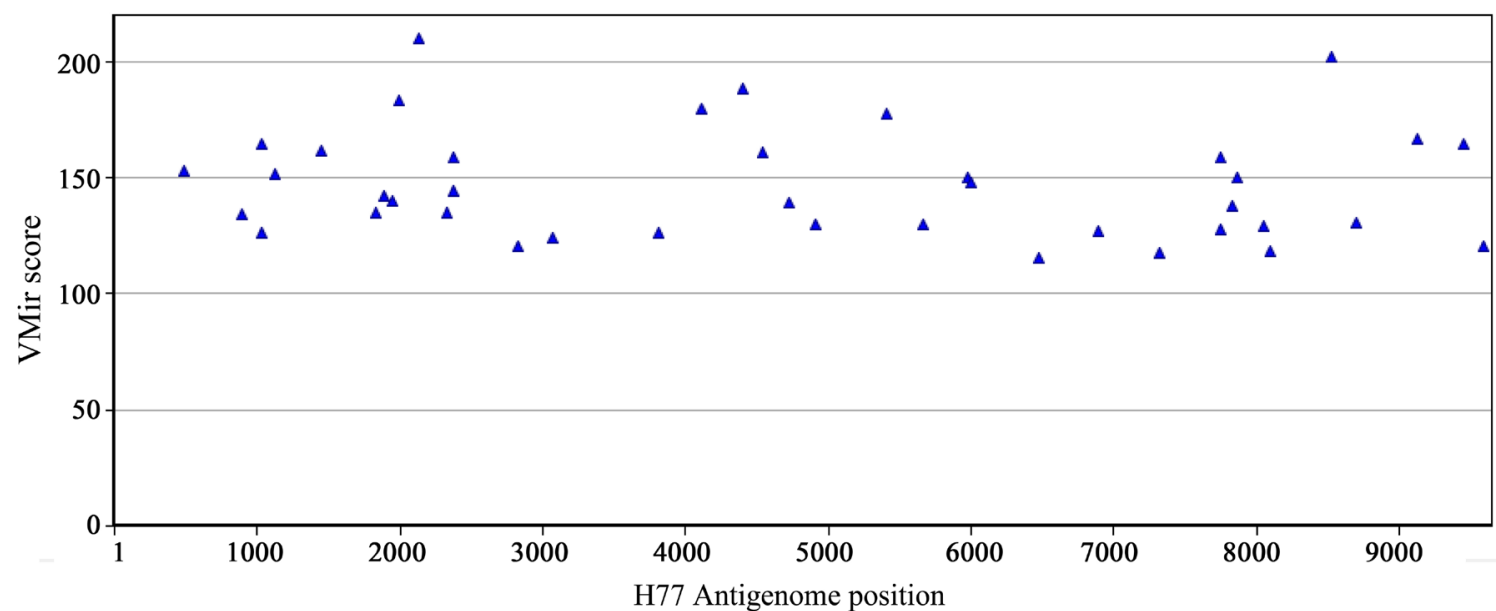

(b)

Figure 2. Graphical view of VMir analysis of the HCV H77 antigenome sequences. In (a) all hairpins of predicted premiRNAs are shown using default settings. In (b) a customized view of predicted pre-miRNA after stringent conditions (minimum hairpin size: $60 \mathrm{nt}$, maximum hairpin size: $120 \mathrm{nt}$, minimum hairpin score: 115, and minimum window count: 25) is shown.

them into real or pseudo pre-miRNAs. Ten different pre-miRNA structures identified by VMir, nominated MD11, MD36, MD19, MD18, MD3, MD39, MD30, MD1, MD26 and MD14, were classified as real pre-miRNAs (data not shown). These structures are present in different different HCV genomic regions (see Table 1).

\subsection{Mature miRNAs}

Comparable to other RNA viruses previously studied, HCV H77 strain miRNAs can be located on either of the two arms in the secondary hairpin structure (see Table 1). Of the ten miRNAs identified in the antigenome of HCV H77 strain, four are located in the 5'-arm of the stem loop hairpin structure, while six are in the 3'-arm (Table 1).

\subsection{Mature miRNAs Sequence Conservation among Different HCV Types and Sub-Types}

HCV presents a high degree of genetic variability. The high error rate of RNA-dependent RNA polymerase and the pressure exerted by the host immune system, has driven the evolution of HCV into 7 different genotypes and a growing number of subtypes. In order to observe if mature miRNA sequences present in the predicted miRNAs found in the antigenome of HCV H77 strain are conserved among different HCV types and subtypes, we search for the similarity among that sequences and corresponding sequences of HCV strains included in a cu- 
Table 1. Predicted hairpin and mature miRNAs sequences within HCV H77 strain antigenome.

\begin{tabular}{cccccc}
\hline Hairpin & Position $^{\boldsymbol{a}}$ & Region $^{\boldsymbol{b}}$ & Score $^{c}$ & Mature 5’arm & Mature 3’arm \\
\hline MD11 & $2086-2179$ & NS5A & 209.6 & AUCCGGAUCCCCAGGCUCCCCC & AAGAAUAGGACUCAACGUCGGA \\
MD36 & $8468-8563$ & E1 & 201.7 & GCUCCCGACAAGCAGAUCGAUA & GGGGAGUUUGCCGUCCCUGGUG \\
MD19 & $4340-4458$ & E2 & 188.2 & GUGAUUGGGUGCGUCAGGGUGA & UGUAUAGCAGGGGUGUUGGCCC \\
MD18 & $4062-4146$ & NS4B & 179.5 & GGGACGCGGUCUGCAGGAGGCC & UGAACUGCUCAGCGAGCAUCAU \\
MD3 & $968-1086$ & NS5B & 164.8 & GGCGGAGUACCUGGUCAUAGCC & GCCGCGUCCUCCUGGACCCCCG \\
MD39 & $9406-9490$ & 5 '-UTR & 164.2 & UCCAGGCAUUGAGCGGGUUUAU & CAAUUCCGGUGUACUCACCG \\
MD30 & $7705-7783$ & E2 & 158.5 & CUGUAGGUAGGCGCGCCCGACC & CCACGGGGCUGGGAGUGAAGC \\
MD1 & $432-533$ & NS5B & 152.9 & UUGAAGAGGUACUUGCCACAUA & CUCUGGACAGAAGCCUAGCGCG \\
MD26 & $5952-6053$ & NS3 & 148.1 & AAGGUCUUGGUCCACAUUGGUA & CUUGGGUGAUGCGAUGGUCCUC \\
MD14 & $2348-2414$ & NS5A & 144.5 & UAGCGGGCAGCCAUGGACCACA & AGGCUUUUUCCACGUCUCUAC \\
\hline
\end{tabular}

${ }^{a}$ Positions relative to HCV strain H77 antigenome. ${ }^{b}$ The region of the HCV genome is indicated. ${ }^{c}$ The VMir score is shown. Mature miRNA sequences are shown in bold.

rated HCV sequence dataset that includes all types and subtypes. The results of these studies revealed that all mature miRNA sequences present in the 10 real pre-miRNA structures found in the antigenome of HCV H77 strain are conserved only among HCV sub-genotype 1a. Interestingly, mature miRNA sequences present in pre-miRNA-MD39 share 100\% similarity among HCV strains belonging to genotypes 1a, 1b, 1c, 2a, 2c, 2k, 5, 6 and 7 , and $95 \%$ similarity to genotype $2 \mathrm{a}, 3 \mathrm{a}, 3 \mathrm{~b}, 3 \mathrm{k}$ and 4 (data not shown). This revealed a high degree of conservation of the mature miRNA sequences of this predicted miRNA among HCV strains. In order to observe if this pre-miRNA structure is conserved among HCV genotypes, the secondary structure of pre-miRNA-MD39 was predicted using corresponding sequences of pre-miRNA-MD39 of different HCV types and subtypes. The results of these studies are shown in Figure 3.

As it can be seen in the figure, very similar secondary structures are obtained using HCV strains of all types. This revealed that this structure is conserved among HCV genotypes.

\subsection{Prediction of the Potential Targets for the Predicted miRNA in the Antigenome of HCV and Functional Annotation}

To understand the dynamics between viral miRNAs and their targets is extremely important to understand the complexity of biological regulation and virus-host interaction. In silico prediction of miRNA targets provides a suitable approach for identifying potential target sites based on their complete or partial complementarity with the miRNAs. For these reasons, pairwise comparison of human 3'-UTR gene transcripts involved in six different metabolic pathways and miRNA-MD39 mature sequences were performed. We observed 17 transcript targets for the predicted HCV-miRNA-MD39 (Table 2). These transcripts are mostly involved in apoptosis as well as immune responses pathways of the host cell (Table 2).

Gene ontology is a useful tool for the mining of gene datasets and their functional annotations. In order to gain insight into these matters, we performed a functional enrichment analysis using KEGG pathway maps. A total of 38 genes were identified (Figure 4).

\subsection{Effective Hybridization of Predicted HCV H77 miRNA and 3'UTR Gene Transcript Targets}

In order to reconfirm effective hybridizations among the identified 3'UTR gene transcript targets and HCVmiR-MD39, we observed their hybridization patterns and calculated the minimum free energy of each hybridization. We have used a MFE of $-14 \mathrm{kcal} / \mathrm{mol}$ as a cut-off value for potential miRNA pairing. The results of these studies are shown in Table 3. As it can be seen in the table, effective hybridizations were found in all cases included in this analysis.

\section{Discussion}

miRNAs play critical roles in many biological processes, such as cell growth, tissue differentiation, cell prolife- 
ration, embryonic development, cell proliferation, and apoptosis. As a consequence, their deregulation perturbs gene expression and can have pathological consequences, as evidenced by their involvement in cancer [30]. Recent studies revealed that virus genomes from different virus families encode miRNAs. Viral miRNAs have been identified by both traditional cloning strategy from virus-infected cells and computational prediction [31]. In this study, using bioinformatics approaches, 10 pre-miRNAs structures with high VMir scores [23] and classified as real pre-miRNA using MiPred [24] were identified in the antigenome of HCV reference strain H77 (see Table 1). This is in agreement with recent results that identified novel miRNAs in the antigenome of yet another hepatic and cytoplasmaticRNA virus, i.e. Hepatitis A virus [21]. Moreover, recent studies carried out in Dengue virus revealed that all the predicted miRNAs were encountered in the reverse strand of the viral mRNA, which adds to the importance of the search of structured noncoding RNAs in replication intermediaries [19].

Mature miRNA sequences of all these 10 potential miRNAs are conserved among HCV genotype 1a, to which H77 strain belongs. Nevertheless, only mature miRNA sequences of pre-miRNA-MD39 were found to be conserved when all HCV genotypes and sub-types are considered. This predicted miRNA maps at the 5' UTR of HCV genome, which is the most conserved genomic region among HCV genotypes[31] (see also Table 1). More-
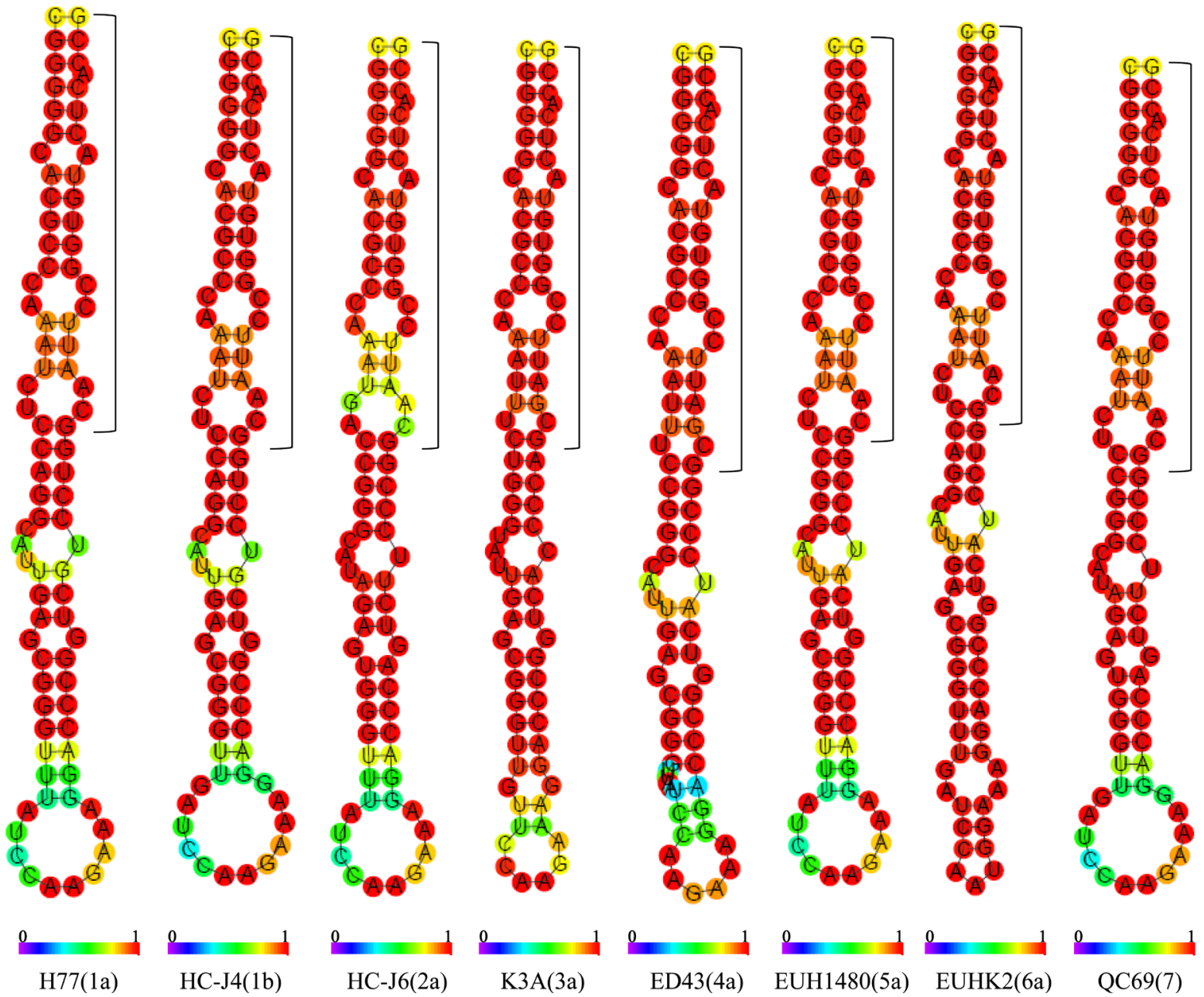

Figure 3. Predicted secondary structure of pre-miRNA-MD39 in different HCV genotypes. The secondary structures predicted for pre-miRNA-MD39 using corresponding sequences of different HCV genotypes and sub-types is shown. Bars at the bottom of the structures denote base pair probabilities. Only centroid structures are depicted.HCV strains are indicated by name and their genotypes are shown between parentheses next to strain name. Mature miRNA sequences are indicated by a square bracket next to each structure. The minimum free energy (MFE) found for the pre-miRNA structures were as follows: $\mathrm{H} 77(1 \mathrm{a})=-35.70 \mathrm{kcal} / \mathrm{mol} ; \mathrm{HC}-\mathrm{J} 4(1 \mathrm{~b})=-35.60 \mathrm{kcal} / \mathrm{mol} ; \mathrm{HC}-\mathrm{J} 6(2 \mathrm{a})=-37.60 \mathrm{kcal} / \mathrm{mol} ; \mathrm{K} 3 \mathrm{~A}(3 \mathrm{a})=-39.90 \mathrm{kcal} / \mathrm{mol}$; $\operatorname{ED} 43(4 \mathrm{a})=-39.90 \mathrm{kcal} / \mathrm{mol} ; \operatorname{EUH} 1480(5 \mathrm{a})=-37.90 \mathrm{kcal} / \mathrm{mol} ; \operatorname{EUHK} 2(6 \mathrm{a})=-41.30 \mathrm{kcal} / \mathrm{mol}$ and QC69(7) $=-35.70$ $\mathrm{kcal} / \mathrm{mol}$. 
Table 2. Predicted HCV H77 antigenome miRNA targets identified by in silico analysis.

\begin{tabular}{|c|c|c|c|c|}
\hline Hairpin & Predicted miRNA & Metabolic pathway & Targeted proteins & Protein description \\
\hline \multirow{17}{*}{ MD39 } & \multirow{17}{*}{ HCV-miR-MD39-3p } & \multirow{3}{*}{$\begin{array}{c}\text { Chemokine signaling } \\
\text { pathway }\end{array}$} & XIAP & X-linked inhibitor of apoptosis \\
\hline & & & GNAI3 & Guanine nucleotide binding protein \\
\hline & & & ITK & IL2-inducible T-cell kinase \\
\hline & & \multirow{3}{*}{$\begin{array}{l}\text { Cytokine-cytokine } \\
\text { receptor interaction }\end{array}$} & LIFR & Leukemia inhibitory factor receptor alpha \\
\hline & & & OSM & Oncostatin M \\
\hline & & & IL21R & Interleukin 21 receptor \\
\hline & & \multirow{5}{*}{$\begin{array}{c}\text { Jak-STAT } \\
\text { signaling pathway }\end{array}$} & CREBBP & CREB binding protein \\
\hline & & & LIFR & Leukemia inhibitory factor receptor alpha \\
\hline & & & OSM & Oncostatin M \\
\hline & & & PIAS3 & Protein inhibitor of activated STAT, 3 \\
\hline & & & IL21R & Interleukin 21 receptor \\
\hline & & \multirow{3}{*}{ mTOR signaling pathway } & PRKAA2 & Protein kinase, AMP-activated, alpha 2 catalytic subunit \\
\hline & & & KIAA1303 & Raptor \\
\hline & & & CAB39L & Calcium binding protein 39-like \\
\hline & & \multirow{3}{*}{$\begin{array}{c}\mathrm{T} \text { cell receptor } \\
\text { signaling pathway }\end{array}$} & CD8A & CD8a molecule \\
\hline & & & ITK & IL2-inducible T-cell kinase \\
\hline & & & ICOS & Inducible T-cell co-stimulator \\
\hline
\end{tabular}

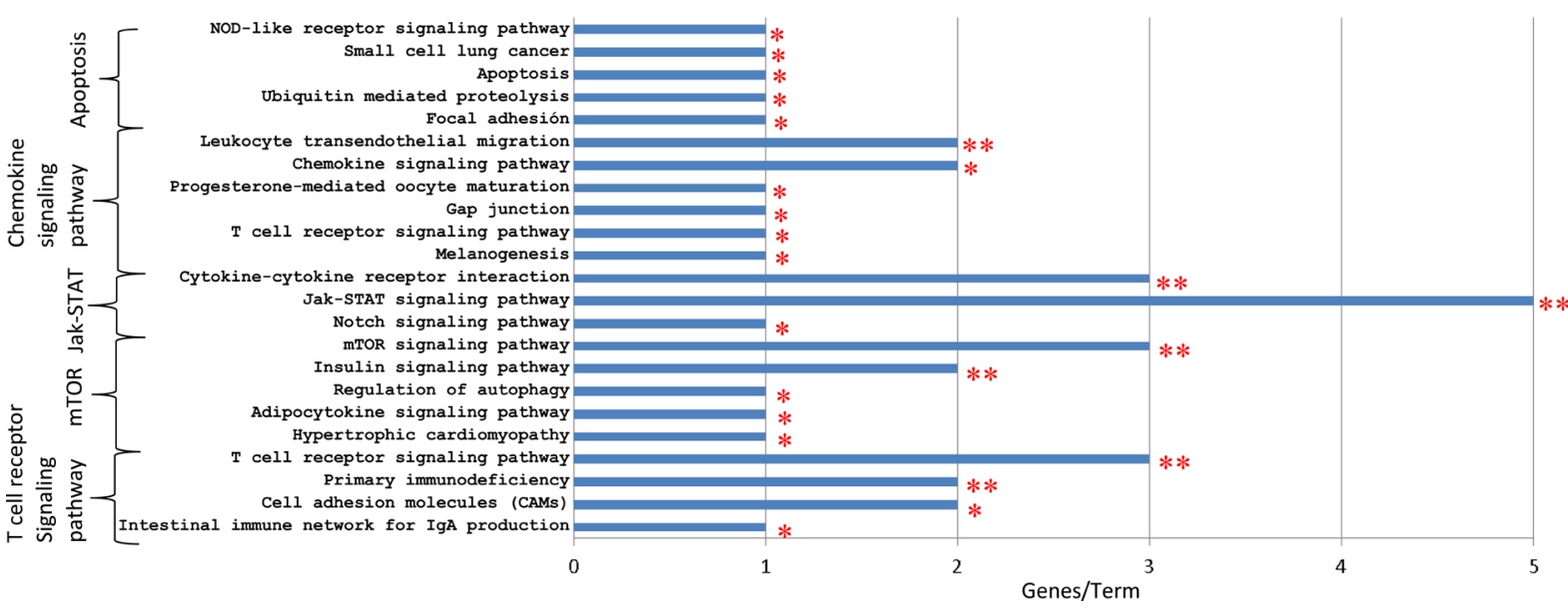

Figure 4. Functional enrichment analysis of the predicted targets of HCV-miR-MD39. Metabolic pathways are shown in the left part of the figure. Bars are proportional to the number of gene/terms in the analysis. *means a $p$-value $<0.05, * *$ means a $p$-value $<0.01$.

over, predictions of the secondary structures of pre-miRNA-MD39 using corresponding sequences of all HCV genotypes and sub-types revealed suitable and similar structures (Figure 3). These studies revealed that this predicted miRNA is conserved among HCV genotypes and may add to the possibility of playing a role in HCV infection.

Recent studies revealed that altered expression of miRNAs is involved in the pathogenesis associated with HCV infection by controlling signaling pathways such as immune response, proliferation and apoptosis [15]. This is in agreement with the results found in this work, since the predicted targets found for HCV-miR-MD39 are members of metabolic pathways related to apoptosis and immune responses (see Table 2 and Figure 4). Moreover, effective hybridizations among the 3'UTR of target gene transcripts and HCV-miR-MD39 were observed in all cases (Table 3).

Taking all together, the results of this work revealed a candidate miRNAs that should be further confirmed by experimental analysis. 
Table 3. Hybridization of predicted HCV H77 miRNA and 3' UTR transcript gene targets identified by an in silico analysis.

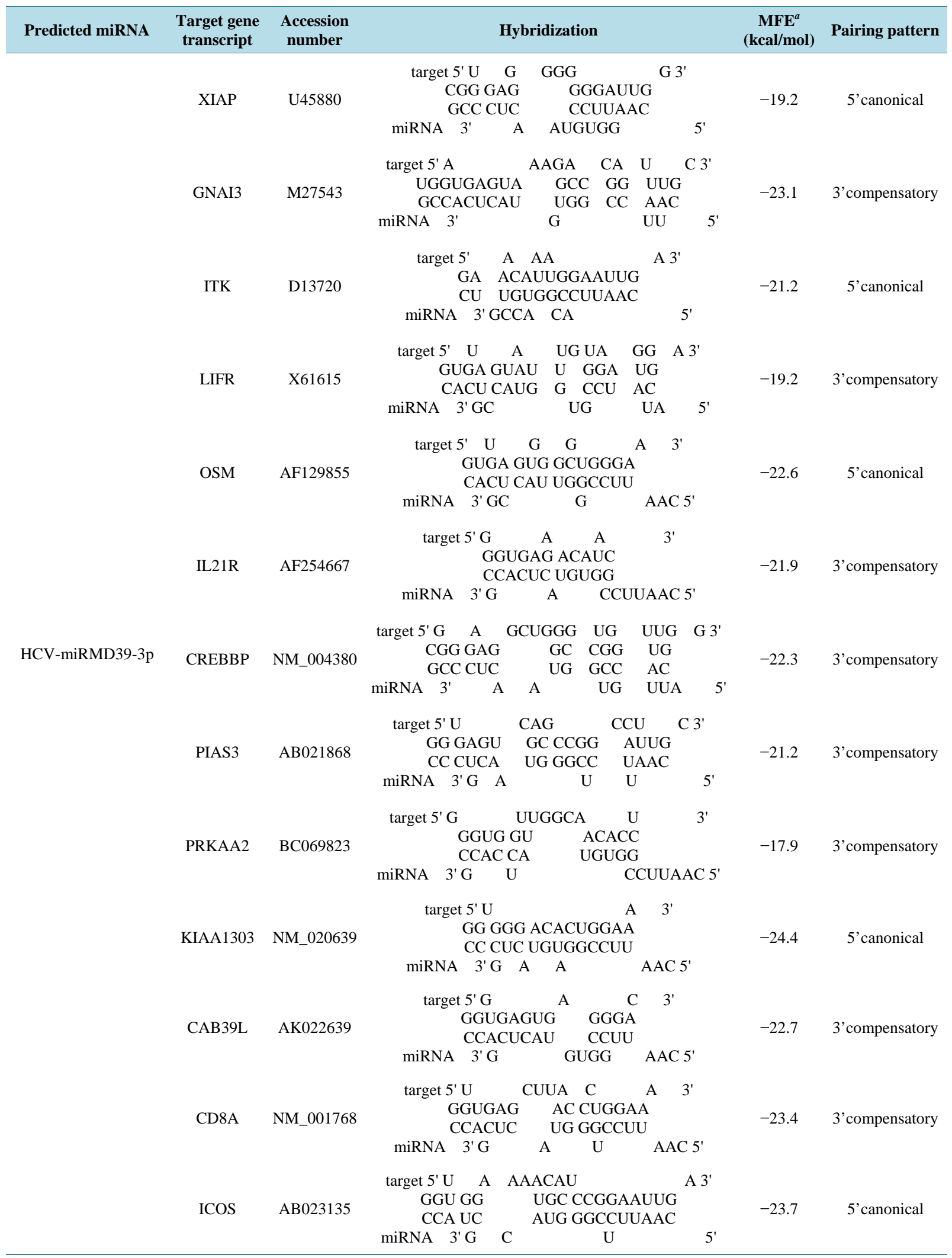

${ }^{a}$ MFE, minimum free energy. 


\begin{tabular}{|c|c|c|c|c|c|c|c|c|}
\hline $\begin{array}{r}3 \\
4 \\
1 \quad 2\end{array}$ & $\begin{array}{l}9 \\
1 \\
5\end{array}$ & $\begin{array}{l}1 \\
4 \\
9 \\
1\end{array}$ & $\begin{array}{ll}2 & 2 \\
5 & 7 \\
8 & 6 \\
0 & 9\end{array}$ & $\begin{array}{l}3 \\
4 \\
2 \\
0\end{array}$ & $\begin{array}{ll}5 & 5 \\
3 & 4 \\
1 & 7 \\
3 & 5\end{array}$ & $\begin{array}{l}6 \\
2 \\
5 \\
8\end{array}$ & $\begin{array}{l}7 \\
6 \\
0 \\
2\end{array}$ & $\begin{array}{l}9 \\
3 \\
7 \\
8\end{array}$ \\
\hline 1 & & & 1 & & 11 & & & \\
\hline
\end{tabular}

Figure 5. The structure of HCV reference strain H77 genomic sequences. The scheme shows the genome of HCV H77 strain. Numbers at the top of the scheme shows nucleotide positions. HCV genes are shown at the bottom. Untranslated regions are indicated at the 5'and 3'end of the genome. Red dot indicates location of the pre-miRNA-MD39 mature sequences.

\section{Conclusions}

Viral miRNAs can be extraordinary important to modulate cellular gene expression. Their small size, high specificity, and capacity for multiple transcript regulation suggest that they could play an important role in virushost interactions during infection. Moreover, a more complex scenario can be seen when the search is extended to include viral replication intermediates.

By utilizing a series of bioinformatics tools, we identified a miRNA present in the antigenome of HCV H77 strain (see Figure 5). This miRNA maps in the 5'UTR region of the HCV genome and is found to be conserved among HCV genotypes and sub-types. In silico target prediction generated 17 cellular genes. These potential targets are involved in apoptosis as well as immune response pathways, suggesting that they could play a role in the pathogenesis caused by viral infection. Besides, the results of these studies revealed the presence of a viral miRNA in the negative-sense RNA strand used as a replication template for the HCV genome, as observed for other RNA viruses [19] [21]. This in silico prediction is a useful guide to experimental design in order to achieve biological validation.

\section{Acknowledgements}

We acknowledge support by Agencia Nacional de Investigación e Innovación (ANII) through project PE_ ALI_ 2009_1_1603, Fondo María Viñas and PhD scholarship program and PEDECIBA, Uruguay.

\section{Conflict of Interest}

The authors declare that there is no conflict of interest regarding the publication of this paper.

\section{References}

[1] Lemon, S.M., Walker, C.M., Alter, M.J. and Yi, M. (2007) Hepatitis C Virus. In: Knipe, D.M. and Howley, P.M., Eds., Fields Virology, Vol. 1, Lippincott Williams \& Wilkins, Philadelphia, 1253-1304.

[2] Alter, M.J. (2007) Epidemiology of Hepatitis C Virus Infection. World Journal of Gastroenterology, 13, $2436-2441$. http://dx.doi.org/10.3748/wjg.v13.i17.2436

[3] Lavanchy, D. (2011) Evolving Epidemiology of Hepatitis C Virus. Clinical and Microbiology Infection, 17, $107-115$. http://dx.doi.org/10.1111/j.1469-0691.2010.03432.x

[4] Farci, P., Alter, H.J., Wong, D., et al. (1991) A Long-Term Study of Hepatitis C Virus Replication in Non-A, Non-B Hepatitis. New England Journal of Medicine, 325, 98-104. http://dx.doi.org/10.1056/NEJM199107113250205

[5] Smith, D.B., Bukh, J., Kuiken, C., et al. (2013) Expanded Classification of Hepatitis C Virus into 7 Genotypes and 67 Subtypes, Updated Criteria and Assignment Web Resource. Hepatology, 59, 318-327. http://dx.doi.org/10.1002/hep.26744

[6] Jackowiak, P., Kuls, K., Budzko, L., et al. (2014) Phylogeny and Molecular Evolution of the Hepatitis C Virus. Infection, Genetics and Evolution, 21, 67-82. http://dx.doi.org/10.1016/j.meegid.2013.10.021

[7] Friedman, R.C., Farh, K.K., Burge, C.B., et al. (2009) Most Mammalian mRNAs Are Conserved Targets of microRNAs. Genome Research, 19, 19-92

[8] Bartel, D.P. (2009) MicroRNAs: Target Recognition and Regulatory Functions. Cell, 136, 215-233. http://dx.doi.org/10.1016/j.cell.2009.01.002

[9] Ambros, V. (2001) microRNAs: Tiny Regulators with Great Potential. Cell, 107, 823-826. http://dx.doi.org/10.1016/S0092-8674(01)00616-X 
[10] Sullivan, C.S. and Ganem, D. (2005) MicroRNAs and Viral Infection. Molecular Cell, 20, 3-7. http://dx.doi.org/10.1016/j.molcel.2005.09.012

[11] Krol, J., Loedige, I. and Filipowicz, W. (2010) The Widespread Regulation of MicroRNA Biogenesis, Function and Decay. Nature Review Genetics, 11, 567-610. http://dx.doi.org/10.1038/nrg2843

[12] Ha, M. and Kim, V.N. (2014) Regulation of MicroRNA Biogenesis. Nature Review Molecular Cell Biology, 15, 509525. http://dx.doi.org/10.1038/nrm3838

[13] Kumar, A. (2011) MicroRNA in HCV Infection and Liver Cancer. Biochimica et Biophysica Acta, 1809, 694-699. http://dx.doi.org/10.1016/j.bbagrm.2011.07.010

[14] Plakunmonthon, S., T-Thienprasert, N.P., Khongnomnan, K., Poovorawan, Y. and Payungporn, S. (2014) Computational Prediction of Hybridization Patterns between Hepatitis C Viral Genome and Human MicroRNAs. Journal of Computational Science, 5, 327-331. http://dx.doi.org/10.1016/j.jocs.2013.12.005

[15] Shrivastava, S., Steele, R., Ray, R. and Ray, R.B. (2015) MicroRNAs: Role in Hepatitis C Virus Pathogenesis. Genes \& Diseases, 2, 35-45. http://dx.doi.org/10.1016/j.gendis.2015.01.001

[16] Zhang, Y., Fan, M., Geng, G., Liu, B., Huang, Z., Luo, H., et al. (2014) A Novel HIV-1-Encoded MicroRNA Enhances Its Viral Replication by Targeting the TATA Box Region. Retrovirology, 11, 23. http://dx.doi.org/10.1186/1742-4690-11-23

[17] Rosewick, N., Momont, M., Durkin, K., Takeda, H., Caiment, F., Cleuter, Y., et al. (2013) Deep Sequencing Reveals Abundant Noncanonical Retroviral MicroRNAs in B-Cell Leukemia/Lymphoma. Proceedings of the National Academy of Sciences of the United States of America, 110, 2306-2311. http://dx.doi.org/10.1073/pnas.1213842110

[18] Hussainand, M. and Asgari, S. (2014) MicroRNA-Like Viral Small RNA from Dengue Virus 2 Autoregulates Its Replication in Mosquito Cells. Proceedings of the National Academy of Sciences of the United States of America, 11, 2746-2751. http://dx.doi.org/10.1073/pnas.1320123111

[19] Ospina-Bedoya, M., Campillo-Pedroza, N., Franco-Salazar, J.P. and Gallego-Gómez, J.C. (2014) Computational Identification of Dengue Virus MicroRNA-Like Structures and Their Cellular Targets. Bioinformatics and Biology Insights, 8, 169-176. http://dx.doi.org/10.4137/BBI.S13649

[20] Hasan, M.M., Akter, R., Ullah, M.S., Abedin, M.J., Ullah, G.M.A. and Hossain, M.Z. (2014) Computational Approach for Predicting Role of Human MicroRNAs in MERS-CoV Genome. Advances in Bioinformatics, 2014, Article ID: 967946. http://dx.doi.org/10.1155/2014/967946

[21] Shi, J., Duan, Z., Sun, J., Wu, M., Wang, B., Zhang, J., et al. (2014) Identification and Validation of a Novel MicroRNA-Like Molecule Derived from a Cytoplasmic RNA Virus Antigenome by Bioinformatics and Experimental Approaches. Virology Journal, 11, 121. http://www.virologyj.com/content/11/1/121 http://dx.doi.org/10.1186/1743-422x-11-121

[22] Tamura, K., Peterson, D., Peterson, N., Stecher, G., Nei, M. and Kumar, S. (2011) MEGA5: Molecular Evolutionary Genetics Analysis Using Maximum Likelihood, Evolutionary Distance, and Maximum Parsimony Method. Molecular Biology and Evolution, 28, 2731-2739. http://dx.doi.org/10.1093/molbev/msr121

[23] Grundhoff, A. (2011) Computational Prediction of Viral miRNAs. In: van Rij, R.P., Ed., Antiviral RNAi: Concepts, Methods, and Applications, Humana Press, New York, 143-152. http://dx.doi.org/10.1007/978-1-61779-037-9_8

[24] Jiang, P., Wu, H., Wang, W., Ma, W., Sun, X. and Lu, Z. (2007) MiPred: Classification of Real and Pseudo MicroRNA Precursors Using Random Forest Prediction Model with Combined Features. Nucleic Acids Research, 35, W339W344. http://dx.doi.org/10.1093/nar/gkm368

[25] Xuan, P., Guo, M., Huang, Y., Li, W. and Huang, Y. (2011) MaturePred: Efficient Identification of MicroRNAs within Novel Plant Pre-miRNAs. PLoS ONE, 6, e27422. http://dx.doi.org/10.1371/journal.pone.0027422

[26] Kuiken, C., Yusim, K., Boykin, L. and Richardson, R. (2005) The Los Alamos HCV Sequence Database. Bioinformatics, 21, 379-384. http://dx.doi.org/10.1093/bioinformatics/bth485

[27] Gruber, A.R., Lorenz, R., Bernhart, S.H., Neubock, R. and Hofacker, I.L. (2008) The Vienna RNA Websuite. Nucleic Acids Research, 36, W70-W74. http://dx.doi.org/10.1093/nar/gkn188

[28] Hsu, J.B., Chiu, C.M., Hsu, S.D., Huang, W.-Y., Chien, C.-H., Lee, T.-Y. and Huang, H.-D. (2011) miRTar: An Integrated System for Identifying miRNA-Target Interactions in Human. BMC Bioinformatics, 12, 300. http://dx.doi.org/10.1186/1471-2105-12-300

[29] Kruger, J. and Rehmsmeier, M. (2006) RNAhybrid: MicroRNA Target Prediction Easy, Fast and Flexible. Nucleic Acids Research, 34, W451-W454. http://dx.doi.org/10.1093/nar/gkl243

[30] Lyra-González, I., Flores-Fong, L.E., González-García, I., Medina-Preciado, D. and Armendáriz-Borunda, J. (2015) MicroRNAs Dysregulation in Hepatocellular Carcinoma: Insights in Genomic Medicine. World Journal of Hepatology, 7, 1530-1540. http://dx.doi.org/10.4254/wjh.v7.i11.1530 
[31] Cui, C., Griffiths, A., Li, G., Silva, L.M., Kramer, M.F., Gaasterland, T., et al. (2006) Prediction and Identification of Herpes Simplex Virus 1-Encoded MicroRNAs. Journal of Virology, 80, 5499-5508.

http://dx.doi.org/10.1128/JVI.00200-06

\section{Abbreviations}

HCV: Hepatitis C Virus

miRNA: MicroRNA 